\title{
Genetically Encoded Inverse Bolaamphiphiles
}

Md Shahadat Hossain, Xin Liu, Timothy I. Maynard, and Davoud Mozhdehi Department of Chemistry, Syracuse University, Syracuse, New York. 13244

Introduction

Post-translational modification of
proteins is widely used in biologica systems and plays an important role in modifying the function of polypeptides. The eukaryotic N-myristoyl transferase (NMT) genetically incorporates myristic acid at the N-terminus of proteins. The
C-terminal domain of a hedgehog C-terminal domain of a hedgehog
protein (HhC) can attach a cholesterol polypeptide by cleaving itself.

Elastin-like-polypeptides (ELP) are class of proteins with rapidly increasing importance due to their unique
properties. By having a lower critical solution temperature (LCST), they
exhibit temperature hierarchical self-assembly.

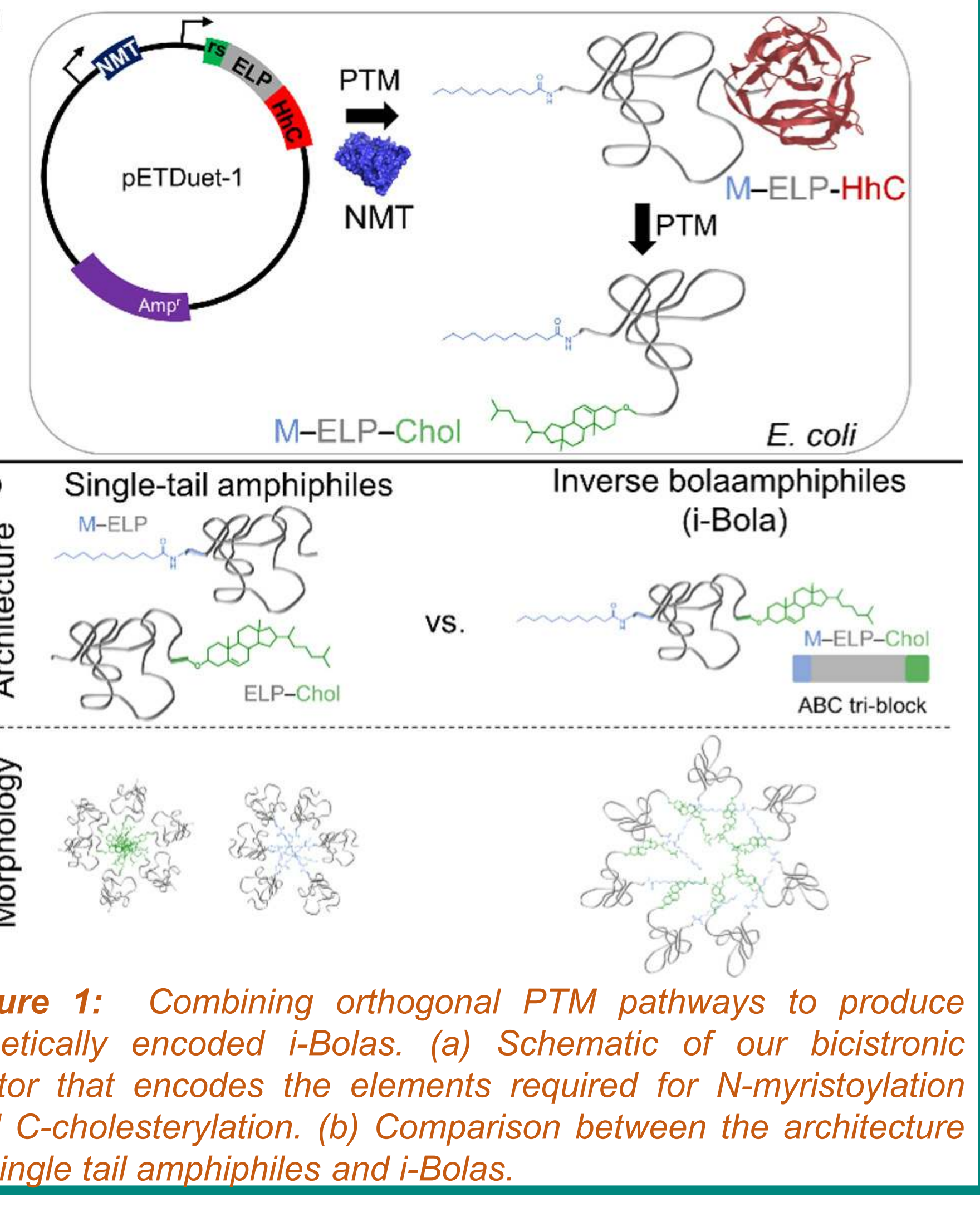

\section{Overview}

We first demonstrated the feasibility of our approach to produce inverse bolaamphiphiles (i-bola), a hydrophlic domain llanked by two hydrophobic domains, respectively. To understand the effect of these tandem PTMs, we then investigated the self assembly of the $\mathrm{i}$-Bolas and compared them to single-tail amphiphile controls bearing
either the $\mathrm{N}$ - or C-terminal lipid using turbidimetry, light scattering, and transmission electron microscopy (TEM).

Finally, we established that these two PTMs can be combined in a prokaryotic expression system to genetically synthesize sequence-defined i-Bolas with high yield and facile purification

These genetic i-Bola structures exhibit hierarchical self-assembly that is significantly promise of our approach to repurpose biological machinery to develop unexplored lipoproteins with tunable nanoscale structures and morphologies.

\section{In vitro Synthesis of i-Bola}

NMT and ELP-HhC were co-expressed from a bicistronic vector inside E. coli. The co-expressed NMT is active and (ELP-HhC) during expression.

Myristoylated ELP-HhC reacts with cholesterol to form two new products, indicating that the $\mathrm{N}$-myristoylation does not interfere with the the hedgehog domain.

Constructs were purified by taking triggered phase transition using inverse transition cycling (ITC), followed by preparative HPLC.

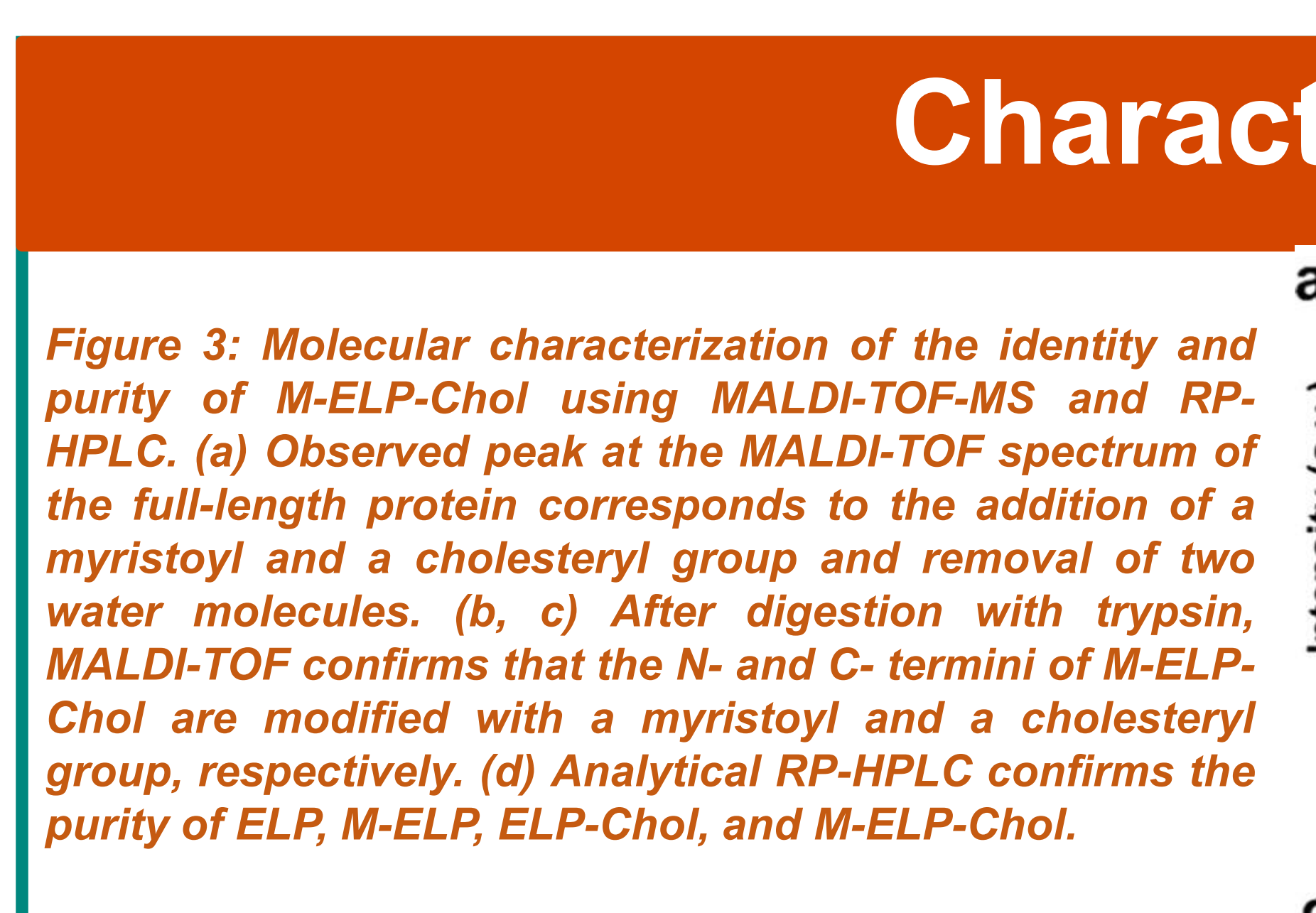

The LCST phase transition was quantified by measuring the turbidity at $350 \mathrm{~nm}$ at different temperatures (Figure 4).

Turbidimetry confirms that lipidation reduces the transition temperature of ELPs. However, the identity and the topology of the lipidation influence how turbidity changes with temperature and molecular parameters ing that these density or coarsening of the ELP coacervates.

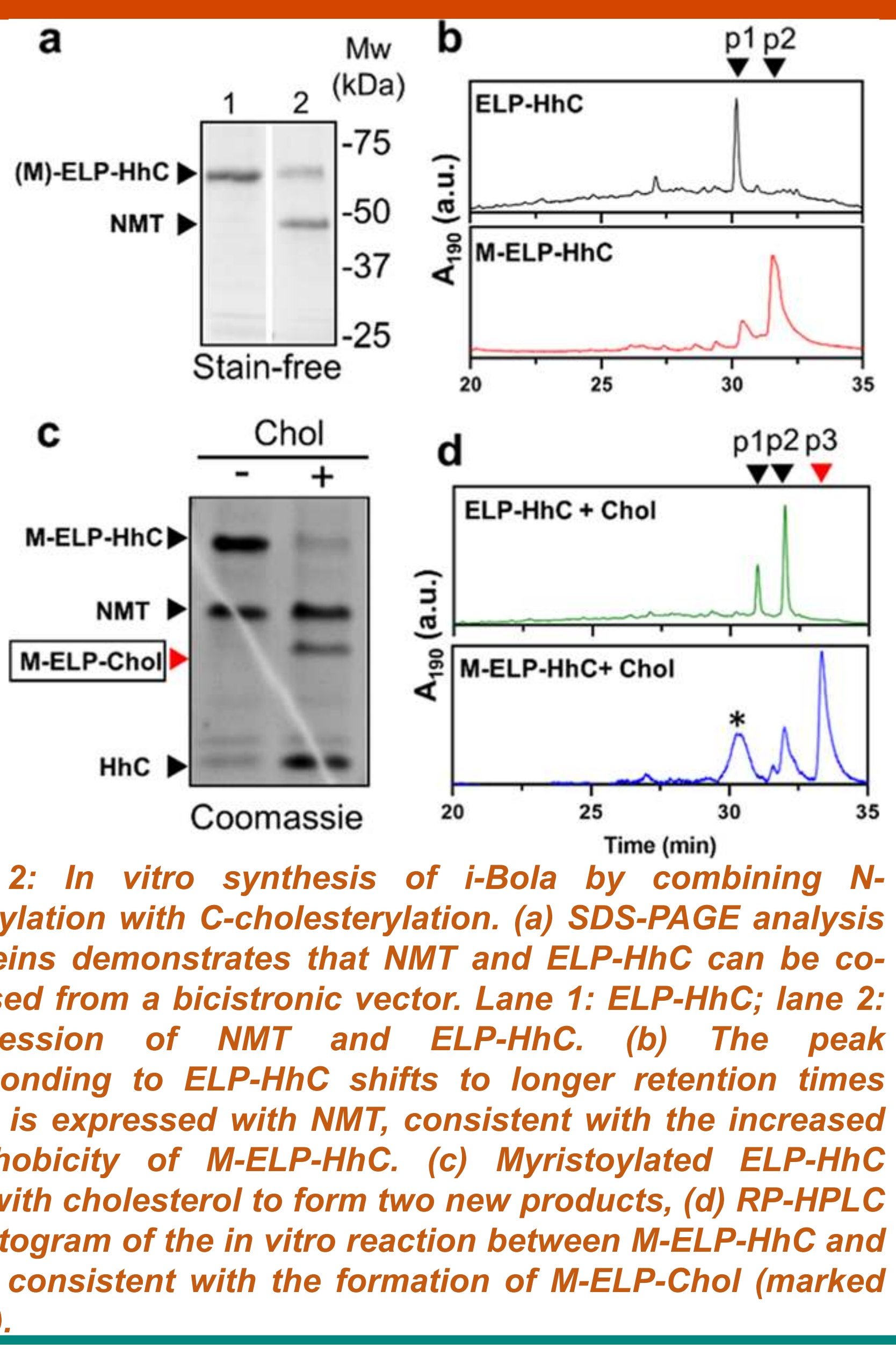

analyzed by TEM.

M-ELP-Chol features larger, noticeably different spherical structures

The result demonstrate that PTM is a powerful approach to control the materials.

- It also confirms our hypothesis that the architecture of lipidated protein (single-tail vs $\mathrm{i}$-Bola) influences the
Application of our Method with Different

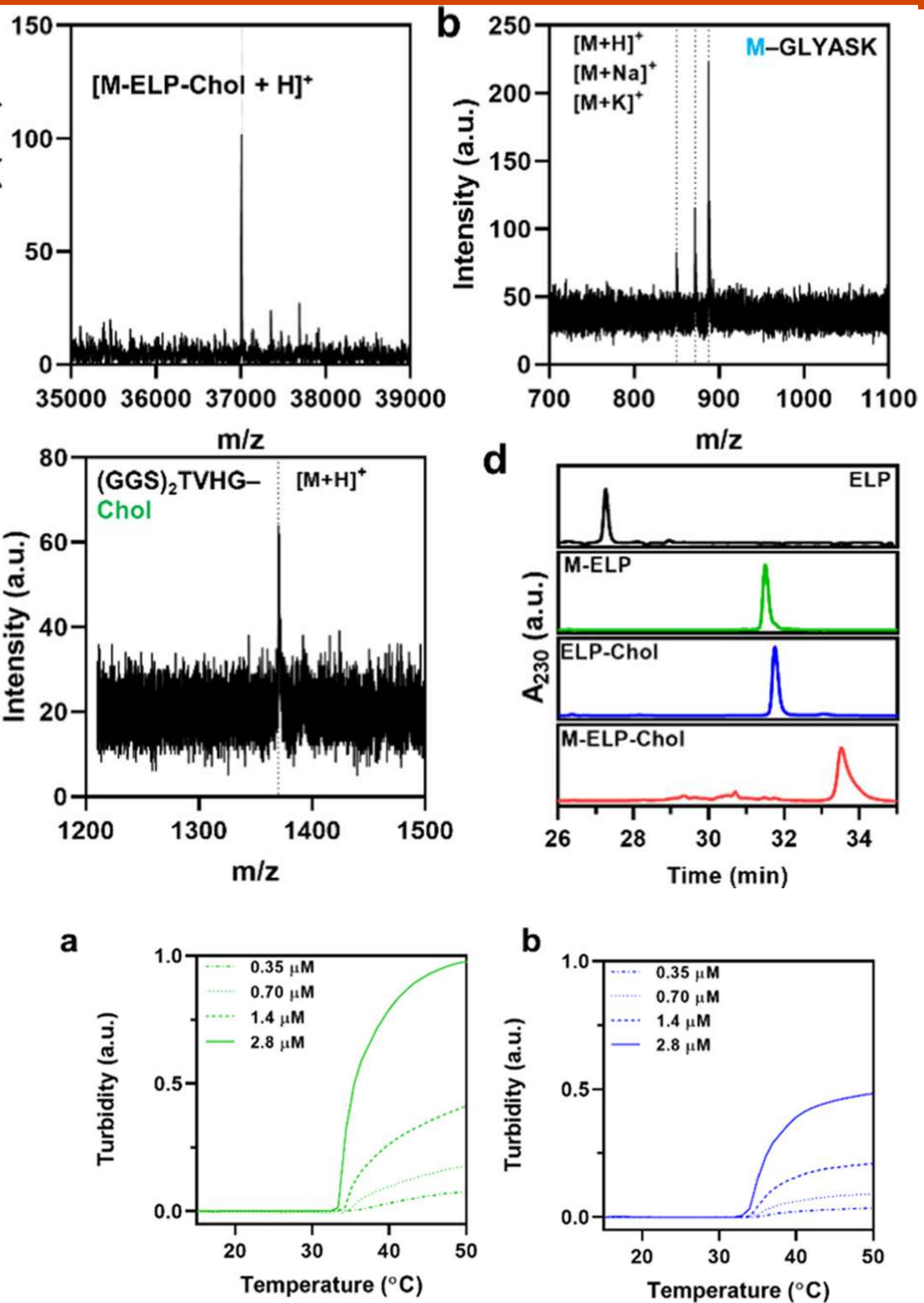

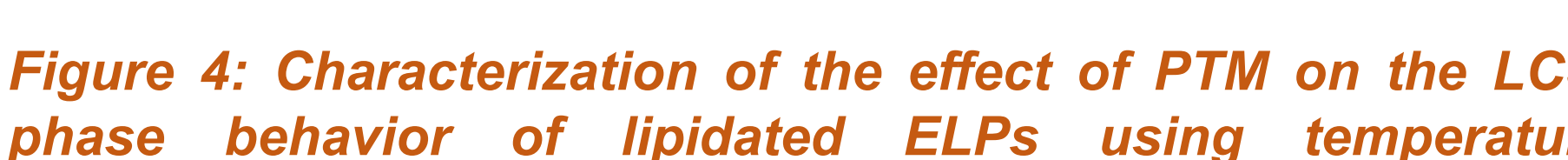

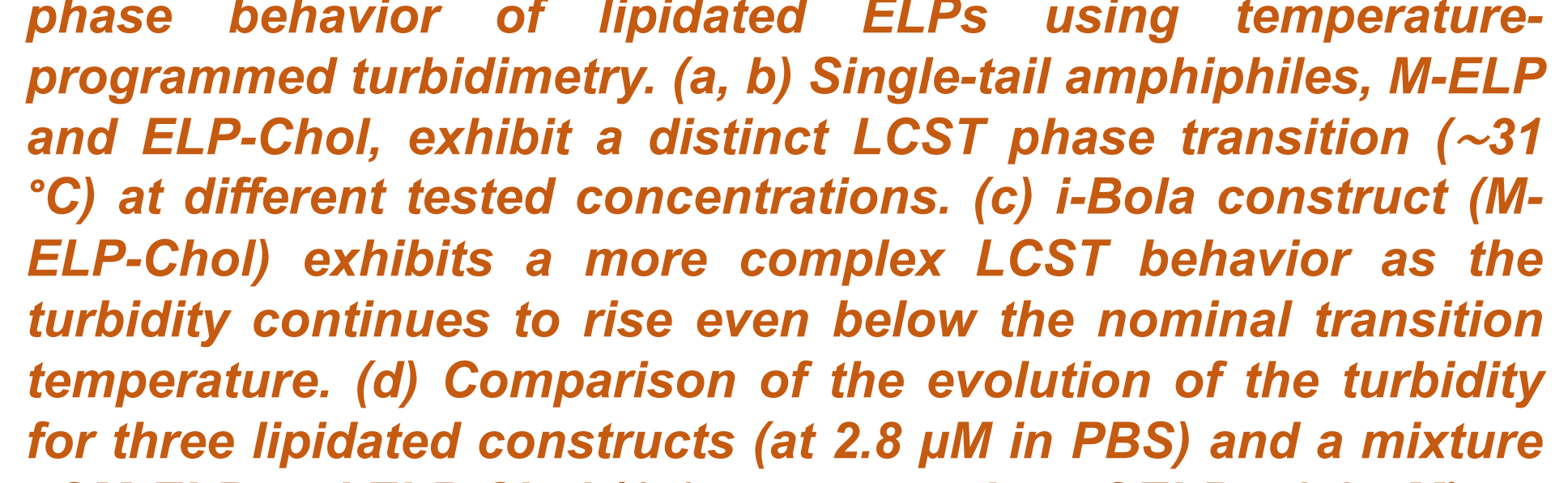
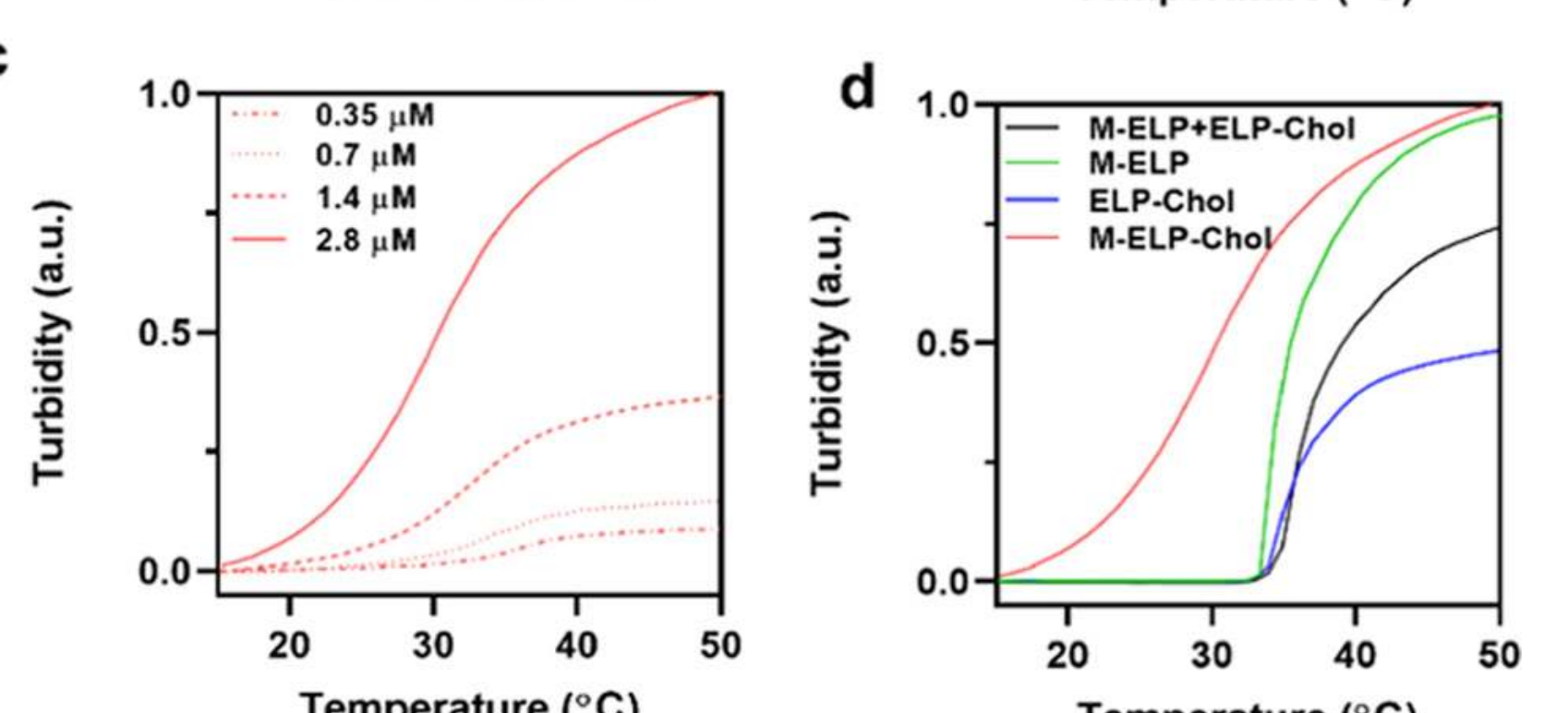

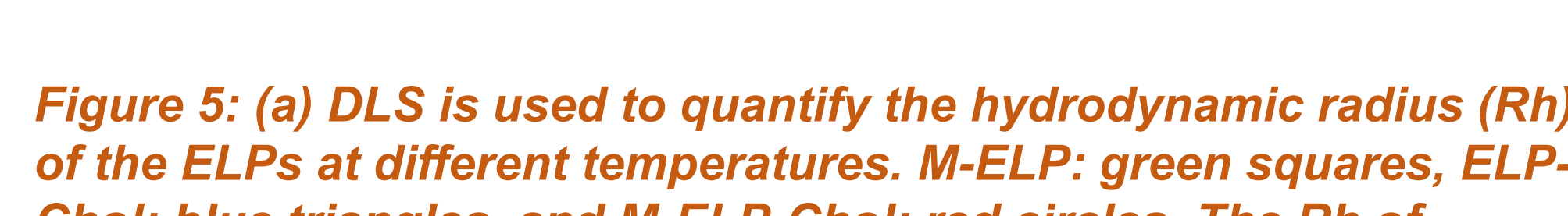

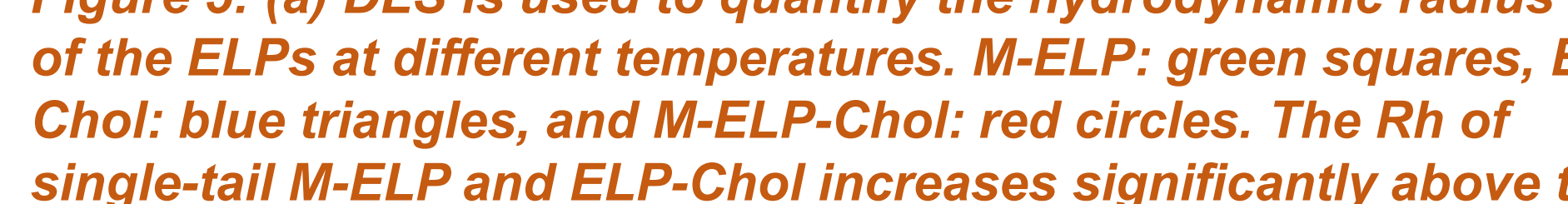

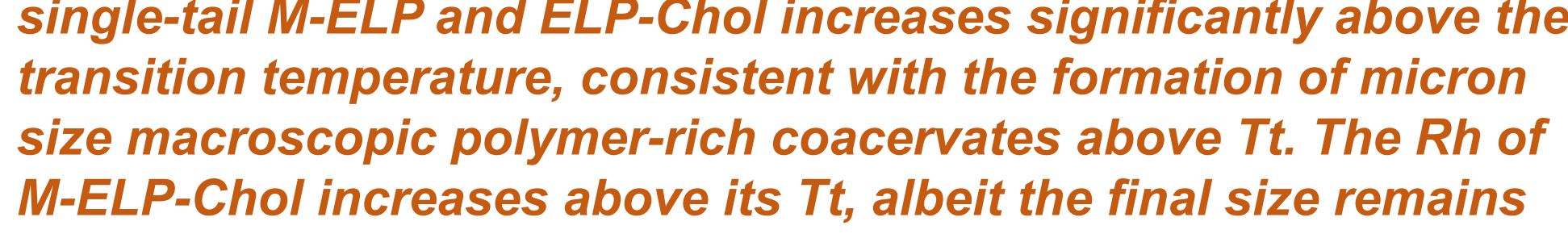

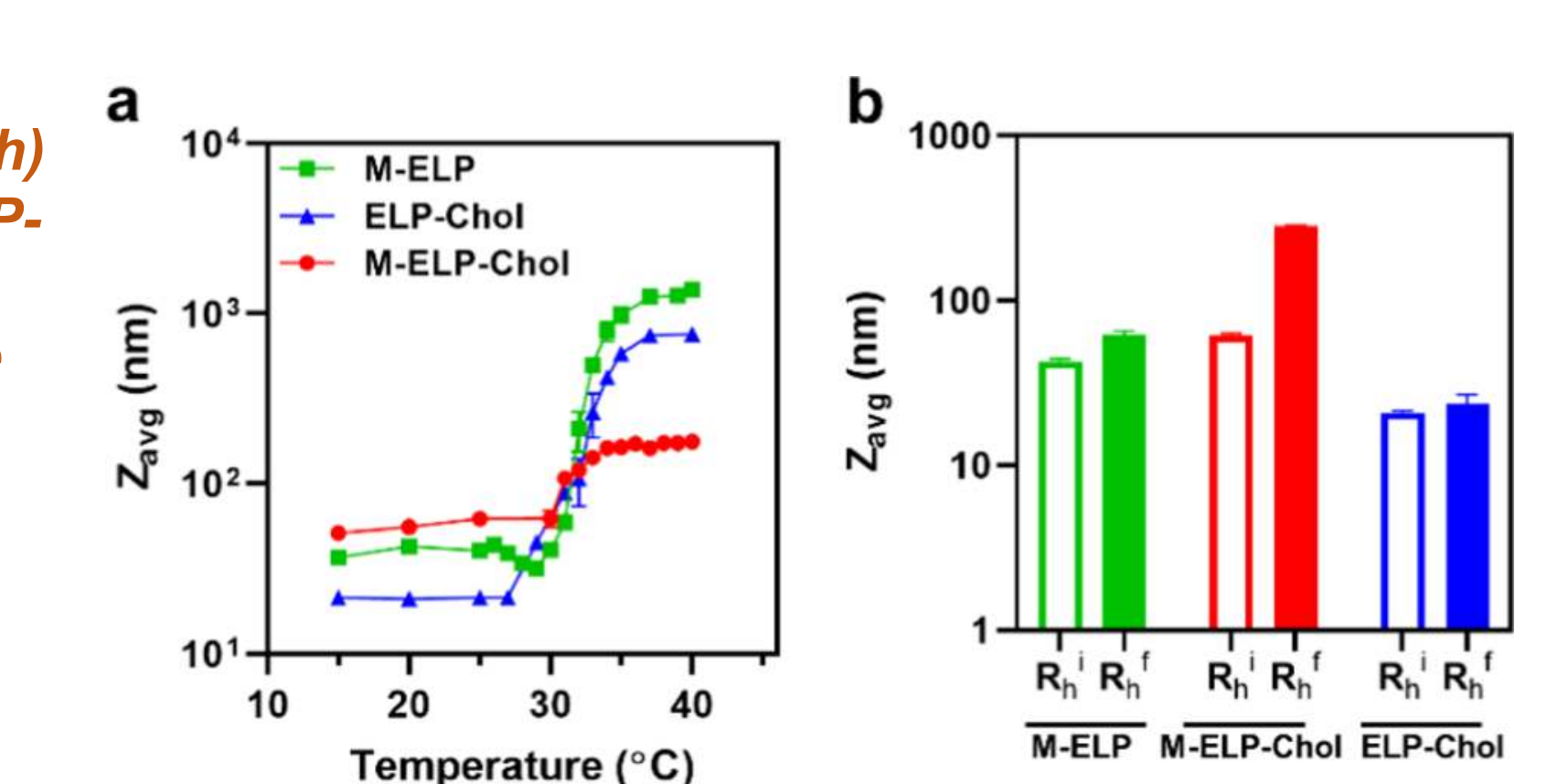

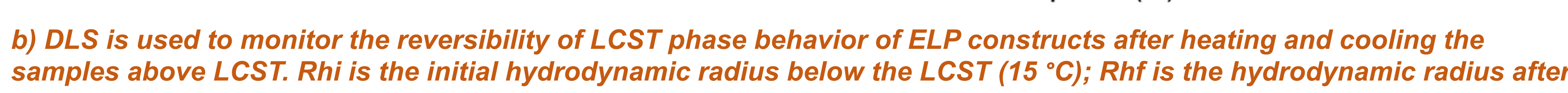

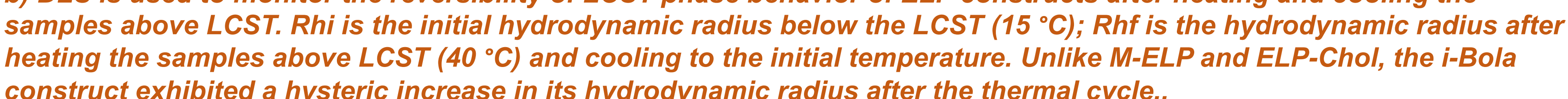

Morphological Characterization

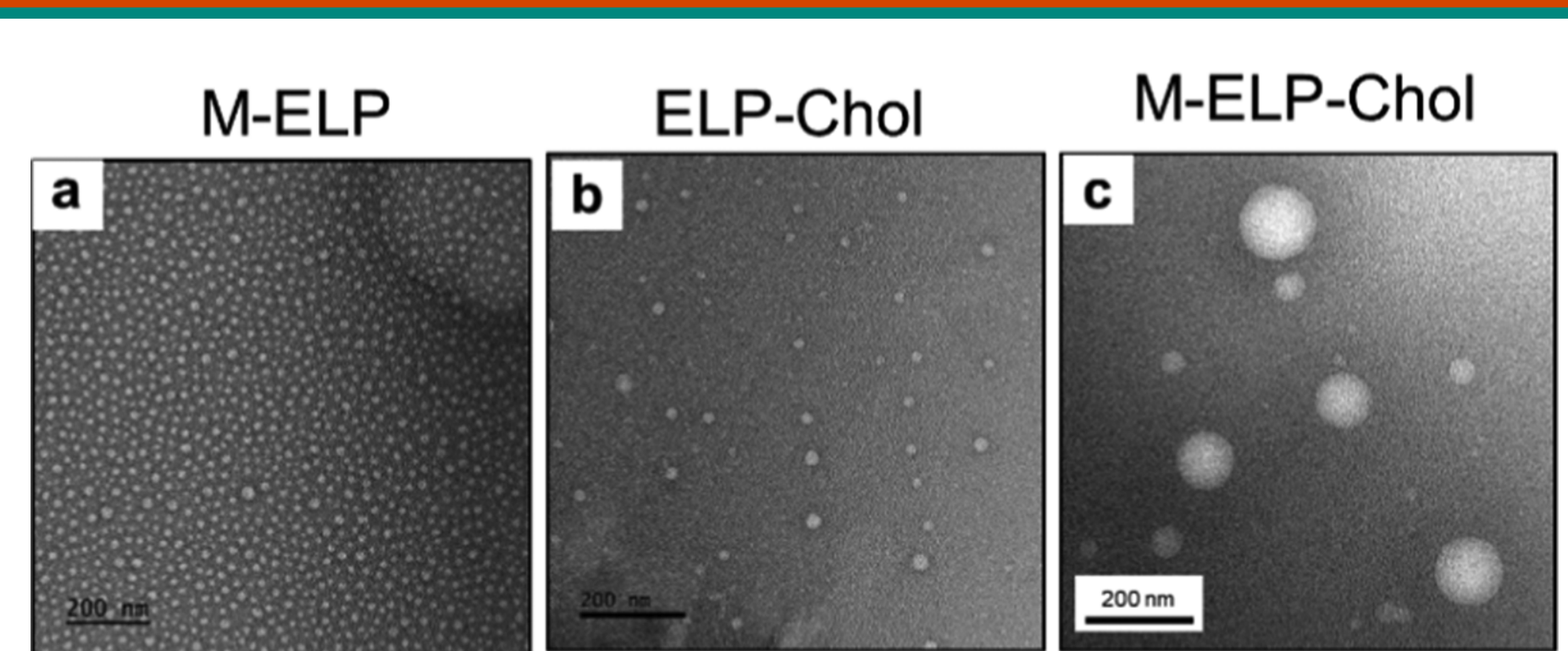
Figure 6: TEM reveals the effect of PTM on the
nanomorphology of proteins in this study. (a) M-ELP and (b)

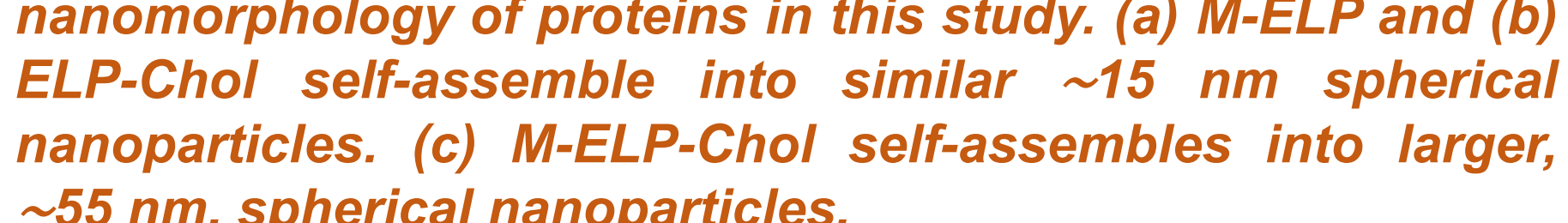
\section{Protein}

We anders our melod to another

- We applied our method to another
construct- (GSGVP) 80 in which the guest

residues were changed from a mixture of
hydrophobic Val and Ala to hydrophilic

hydrophobic Val and
Ser residues (sELP).

- The construct is self-assembled into a mixture of spherical and ellipsoidal particles with an average diameter of 60.3 $\pm 11.4 \mathrm{~nm}$ (Figure $7 \mathrm{c}$ ). This is possibly
due to the lack of repulsive due to the lack of repulive
charged-charged interactions along the polypeptide backbone (as M-sELP-Chol does not contain positively charged lysine
residues that are found in M-ELP-Chol).

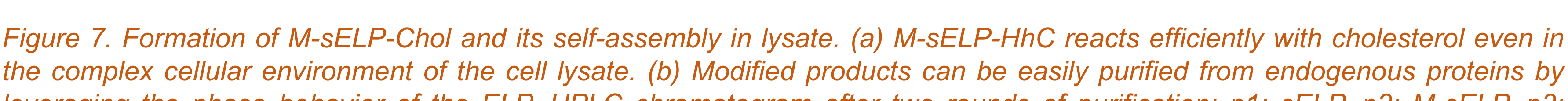

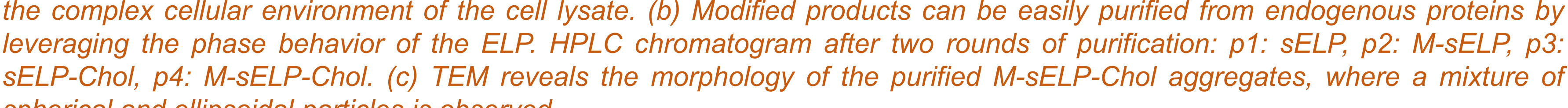

\section{Conclusion}

We have demonstrated for the first time that it is possible to leverage the inherent regioselectivity of the PTM machinery to obtain post-translationally modified polypeptides with i-Bola structure. Our recombinant method provides a facile approach to synthesize protein-based
nanoparticles and expands the phase-space of available nanostructures beyond single-tail amphiphiles.

Our results also indicate that the architecture of lipidated protein and the sequence of the polypeptide can be used to control the hierarchical self-assembly of these protein(a)

The deeper understanding of molecular parameters controlling the assembly of this new class of biomaterials, achieved by the combination of computational and experimental tools, is the critical next step toward advancing their use in applications nanoreactors templating the formation of biominerals.

\section{References}

Hossain, M. S.; Liu, X.; Maynard, T. I.; Mozhdehi, D. Genetically Encoded Inverse Bolaamphiphiles. Biomacromolecules 2020, 21 (2), 660-669.

Sonnenburg, E. D.; Gordon, J. I. Protein N-Myristoylation. In Encyclopedia of Biological Chemistry; Elsevier, 2013; Vol. 266, pp 641-644.

Porter, J. A.; von Kessler, D. P.; Ekker, S. C.; Young, K. E.; Lee, J. J.; Moses, K.; Beachy, P. A. The Product of Hedgehog Autoproteolytic Cleavage Active in Local and Long-Range Signalling. Nature 1995, 374, 363-366.

\section{Acknowledgement}

Startup grant (D.M.) from Syracuse University

Professor Matthew M. Maye for generously providing access to $D L S$ 